\title{
On Equalities Involving Integrals of the Logarithm of the Riemann $\varsigma$-Function with Exponential Weight Which Are Equivalent to the Riemann Hypothesis
}

\author{
Sergey K. Sekatskii, ${ }^{1}$ Stefano Beltraminelli, ${ }^{2}$ and Danilo Merlini ${ }^{2}$ \\ ${ }^{1}$ Laboratoire de Physique de la Matière Vivante, IPSB, BSP 408, Ecole Polytechnique Fédérale de Lausanne, 1015 Lausanne, Switzerland \\ ${ }^{2}$ Research Center for Mathematics and Physics (CERFIM), P.O. Box 1132, 6600 Locarno, Switzerland \\ Correspondence should be addressed to Sergey K. Sekatskii; serguei.sekatski@epfl.ch
}

Received 30 April 2015; Revised 4 September 2015; Accepted 6 September 2015

Academic Editor: Remi Léandre

Copyright ( $\odot 2015$ Sergey K. Sekatskii et al. This is an open access article distributed under the Creative Commons Attribution License, which permits unrestricted use, distribution, and reproduction in any medium, provided the original work is properly cited.

Integral equalities involving integrals of the logarithm of the Riemann $\varsigma$-function with exponential weight functions are introduced, and it is shown that an infinite number of them are equivalent to the Riemann hypothesis. Some of these equalities are tested numerically. The possible contribution of the Riemann function zeroes nonlying on the critical line is rigorously estimated and shown to be extremely small, in particular, smaller than nine milliards of decimals for the maximal possible weight function $\exp (-2 \pi t)$. We also show how certain Fourier transforms of the logarithm of the Riemann zeta-function taken along the real (demi)axis are expressible via elementary functions plus logarithm of the gamma-function and definite integrals thereof, as well as certain sums over trivial and nontrivial Riemann function zeroes.

\section{Introduction}

In recent papers $[1,2]$ we analyzed certain contour integrals involving the logarithm of the Riemann zeta-function and have established an infinite number of equalities of the type $\int_{b-i \infty}^{b+i \infty} g(z) \ln (\varsigma(z)) d z=f(b)$ which were proven to be equivalent to the Riemann hypothesis $(\mathrm{RH} ; \varsigma(z)$ is the Riemann zeta-function; see, e.g., [3] for definitions and discussion of the general properties of this function). In particular, it was shown that all earlier known equalities of this type, that is, those of Wang [4], Volchkov [5], Balazard et al. [6], and one of us [7], are certain particular cases of our general approach elaborated in [1].

In this paper we establish new integral equalities equivalent to RH. We use exponential weight functions, and, in our opinion, the resulting equations are especially interesting. In particular, we were able to rigorously estimate the possible contribution of the Riemann function zeroes nonlying on the critical line which were shown to be extremely small, for example, smalle8r than nine milliards of decimals for the "maximal possible"; in a sense (see below), weight function $g(z)=\exp (-2 \pi z)$.

\section{Integral Equalities with Exponential Weight Function Equivalent to the Riemann Hypothesis}

The main tool for our work here is the following generalized Littlewood theorem about contour integrals involving logarithm of an analytical function.

Theorem 1 (the generalized Littlewood theorem). Let $C$ denote the rectangle bounded by the lines $x=X_{1}, x=X_{2}, y=$ $Y_{1}, y=Y_{2}$ where $X_{1}<X_{2}, Y_{1}<Y_{2}$ and let $f(z)$ be analytic and nonzero on $C$ and meromorphic inside it; let also $g(z)$ be analytic on $C$ and meromorphic inside it. Let $F(z)=\ln (f(z))$ be the logarithm defined as follows: one starts with a particular determination on $x=X_{2}$ and obtains the value at other points by continuous variation along $y=$ const from $\ln \left(X_{2}+i y\right)$. If, however, this path would cross a zero or pole of $f(z)$, one takes 
$F(z)$ to be $F(z \pm i 0)$ accordingly as one approaches the path from above or below. Let also $\widetilde{F}(z)=\ln (f(z))$ be the logarithm defined by continuous variation along any smooth curve fully lying inside the contour which avoids all poles and zeroes of $f(z)$ and starts from the same particular determination on $x=X_{2}$. Suppose also that the poles and zeroes of the functions $f(z), g(z)$ do not coincide.

Then $\int_{C} F(z) g(z) d z=2 \pi i\left(\sum_{\rho_{g}} \operatorname{res}\left(g\left(\rho_{g}\right) \cdot \widetilde{F}\left(\rho_{g}\right)\right)-\right.$ $\left.\sum_{\rho_{f}^{0}} \int_{X_{1}+i Y_{\rho}^{0}}^{X_{\rho}^{0}+i Y_{\rho}^{0}} g(z) d z+\sum_{\rho_{f}^{p o l}} \int_{X_{1}+i Y_{\rho}^{p o l}}^{X_{p}^{p o l}+i Y_{\rho}^{p o l}} g(z) d z\right)$, where the sum is over all $\rho_{g}$ which are poles of the function $g(z)$ lying inside $C$, all $\rho_{f}^{0}=X_{\rho}^{0}+i Y_{\rho}^{0}$ which are zeroes of the function $f(z)$ counted taking into account their multiplicities (i.e., the corresponding term is multiplied by $m$ for a zero of the order $m$ ) and which lie inside $C$, and all $\rho_{f}^{p o l}=X_{\rho}^{p o l}+i Y_{\rho}^{p o l}$ which are poles of the function $f(z)$ counted taking into account their multiplicities and which lie inside C. The assumption is that all relevant integrals in the right-hand side of the equality exist.

Proof. Our proof closely follows the well-known proof of the Littlewood theorem (or lemma) given, for example, in [8, p. 133]. Consider first the function $f(z)=z-a$ where $a=\alpha+i \beta$ is a point of the rectangle. Let $C^{\prime}$ be the contour obtained by describing $C$ in the positive direction from $\left(X_{2}, Y_{1}\right)$ as far as $\left(X_{1}, \beta\right)$, then the straight line $y=\beta$ as far as $\alpha-\varepsilon+i \beta$, then a circle of radius $\varepsilon$ about $z=a$, and then returning along $y=\beta$ and the rest of $C$ to the starting point; see Figure 1 . The only poles of $F(z) g(z)$ in $C^{\prime}$ are those of the function $g(z)$, so that $\int_{C^{\prime}} F(z) g(z) d z=2 \pi i \sum_{\rho_{g}} \operatorname{res}\left(g\left(\rho_{g}\right) \cdot \widetilde{F}\left(\rho_{g}\right)\right)$. The integral round the small circle tends to zero with the radius; thus we have $\int_{C} F(z) g(z) d z=2 \pi i \sum_{\rho_{g}} \operatorname{res}\left(g\left(\rho_{g}\right) \cdot \widetilde{F}\left(\rho_{g}\right)\right)-$ $\int_{X_{1}+i \beta}^{\alpha+i \beta} g(z)\left(F_{1}(z)-F_{2}(z)\right) d z$, where $F_{1}$ and $F_{2}$ are the values of $F$ on the two paths joining $X_{1}+i \beta$ to $\alpha+i \beta$. Hence we obtain $F_{2}$ from $F_{1}$ by passing in the negative direction round a simple zero of $f(z)$ at $z=a$; we have $F_{2}(z)=F_{1}(z)-2 \pi i$ and, correspondingly, $\int_{C} F(z) g(z) d z=2 \pi i\left(\sum_{\rho_{g}} \operatorname{res}\left(g\left(\rho_{g}\right)\right.\right.$. $\left.\left.\widetilde{F}\left(\rho_{g}\right)\right)-\int_{X_{1}+i \beta}^{\alpha+i \beta} g(z) d z\right)$, where we introduce a notation $\widetilde{F}(z)$ to distinguish this function from $F(z)$. The general case now easily follows by addition of terms corresponding to the various poles and zeroes of $f(z)$.

From the proof of Theorem 1, illustrated in Figure 1, it follows that the function $F(z)=\ln (f(z))$, occurring in its formulation, is not a continuous function along the left border of the contour, namely, along the line connecting the points $X_{1}+i Y_{1}$ and $X_{1}+i Y_{2}$ : its value jumps on $\mp 2 \pi l$ when we pass a point $X_{1}+i Y$ such that there is an $l$ th order zero or pole of the function $f(z)$ lying inside the contour (and not on the integration line) and having an ordinate $Y$. This function is only piecewise continuous. This circumstance is present, of course, also in the original Littlewood theorem given for $g(z)=1$ (and is well recognized in its formulation; see [8]). The value of $\ln |f(z)|$ is a continuous function along the left border of the contour provided, of course, that there are no

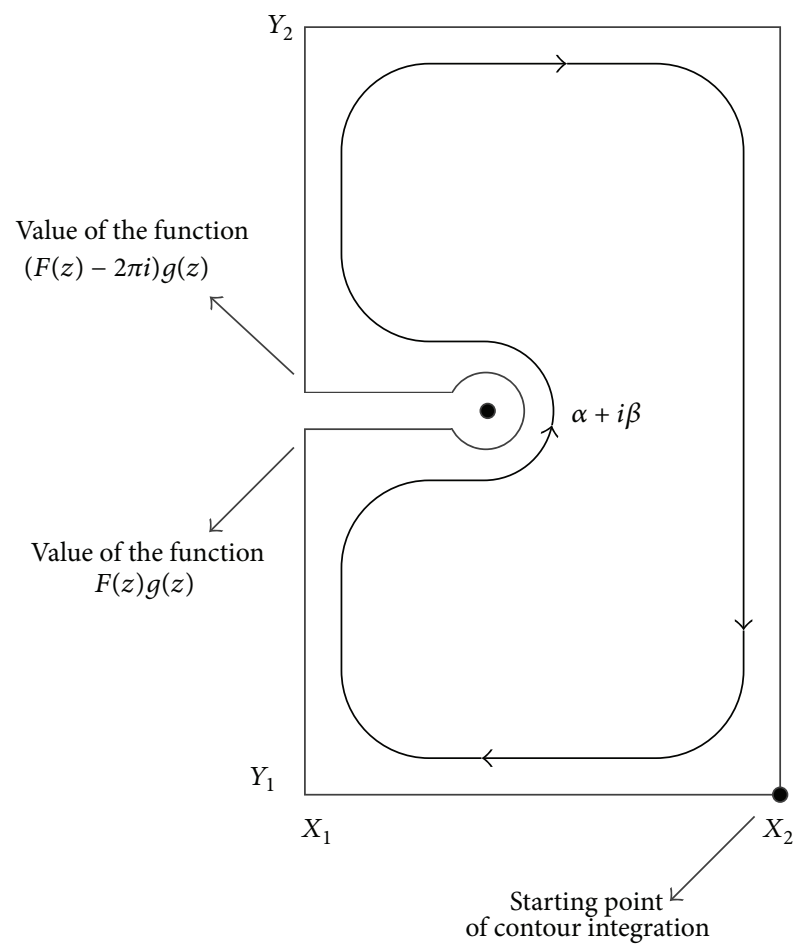

FIGURE 1: Illustrating the proof of the generalized Littlewood theorem.

poles or zeroes of the function $f(z)$ lying exactly on this same border.

Now let us consider a rectangular contour $C$ with the vertices $b, b+i X, b+X+i X, b+X$ with real $b>-2$ (with this choice we avoid the trivial Riemann zeroes) and real $X \rightarrow$ $+\infty$, introduce the function $g(z)=i \exp i(a(z-b))$, where $a$ is real positive, and apply the above theorem to the contour integral $\int_{C} g(z) \ln (\varsigma(z)) d z$. Along the line $(b, b+i X)$ we have an integral $\int_{0}^{X} e^{-a t} \ln ^{*}(\varsigma(b+i t)) d t$ and along the line $(b, b+X)$ we have an integral $i \int_{0}^{X}(\cos (a x)+i \sin (a x)) \ln (\varsigma(b+x)) d x$. Due to the known asymptotic behaviour of the Riemann zeta-function [3], in particular $\ln (\varsigma(z))=O\left(2^{-z}\right)$ for large positive $z$, these integrals converge while integrals over lines $(X, X+i X)$ and $(b+i X, X+i X)$ vanish in the limit of large $X$.

If $b<1$, on the border of the contour, we have the pole of the Riemann function at $z=1$ and in the interior of the contour we also might have a number of zeroes of the Riemann function. We definitely have an infinite number of zeroes if $b<1 / 2$. The order $l_{k}$ zero of the Riemann function $\rho=\sigma_{k}+i t_{k}$, for $b<\sigma_{k}$, according to Theorem 1 , contributes $-2 \pi i l_{k} \int_{0}^{\sigma_{k}-b} e^{-a t_{k}}(i \cos (a x)-\sin (a x)) d x$ $=\left(2 \pi l_{k} e^{-a t_{k}} / a\right)\left[\sin \left(a\left(\sigma_{k}-b\right)\right)+i\left(1-\cos \left(a\left(\sigma_{k}-b\right)\right)\right)\right]$ to the contour integral value, so finally the aforementioned 
generalized Littlewood theorem results in the following equality:

$$
\begin{aligned}
& \int_{0}^{\infty} e^{-a t} \ln ^{*}(\varsigma(b+i t)) d t-\int_{0}^{\infty} \sin (a x) \ln (\varsigma(b+x)) d x \\
& +i \int_{0}^{\infty} \cos (a x) \ln (\varsigma(b+x)) d x=\frac{2 \pi}{a} \\
& \quad \cdot \sum_{\rho, t_{k}>0, \sigma_{k}>b} l_{k} e^{-a t_{k}}\left[\sin \left(a\left(\sigma_{k}-b\right)\right)\right. \\
& \left.+i\left(1-\cos \left(a\left(\sigma_{k}-b\right)\right)\right)\right]-\frac{\pi}{a}(\sin (a(1-b))+i(1 \\
& \quad-\cos (a(1-b)))) .
\end{aligned}
$$

In the last term here we took into account that the pole $z=1$ lies exactly onto the contour border whence the coefficient is $\pi / a$ instead of $2 \pi / a$.

Now we should remind the reader that the function $\ln ^{*}(\varsigma(b+i t))$ is not continuous; see above (and this is the reason why we put an asterisk sign here). If we want to use a continuous logarithm function instead (which we will denote simply $\ln (\varsigma(b+i t)))$, the following straightforward modification is to be done. If real functions $f(x), g(x)$ are continuous on the segment $[A, B]$ and function $f^{*}(x)$ coincides with $f(x)$ on $[A, C]$ and is equal to $f(x)+h$ on $(C, B]$ ( $h$ is a constant, $A \leq C \leq B$ ), we have $\int_{A}^{B} g(x) f^{*}(x) d x=\int_{A}^{B} g(x) f(x) d x+h \int_{C}^{B} g(x) d x$, and this is trivially generalized for complex numbers and improper integrals having counting number of discontinuities. For our particular case we have, considering one $2 \pi i l_{k}$, discontinuity at $t=t_{k}: \int_{0}^{\infty} e^{-a t} \arg ^{*}(\varsigma(b+i t)) d t=\int_{0}^{\infty} e^{-a t} \arg (\varsigma(b+i t)) d t+$ $\left(2 \pi l_{k} / a\right) e^{-a t_{k}}$, and the aforementioned generalization gives

$$
\begin{aligned}
& \int_{0}^{\infty} e^{-a t} \arg ^{*}(\varsigma(b+i t)) d t \\
& \quad=\int_{0}^{\infty} e^{-a t} \arg (\varsigma(b+i t)) d t+\sum_{\rho, t_{k}>0, \sigma_{k}>b} \frac{2 \pi l_{k}}{a} e^{-a t_{k}} .
\end{aligned}
$$

Using (2), we get instead of (1)

$$
\begin{aligned}
& \int_{0}^{\infty} e^{-a t} \ln (\varsigma(b+i t)) d t-\int_{0}^{\infty} \sin (a x) \ln (\varsigma(b+x)) d x \\
& \quad+i \int_{0}^{\infty} \cos (a x) \ln (\varsigma(b+x)) d x=\frac{2 \pi}{a} \\
& \quad \cdot \sum_{\rho, t_{k}>0, \sigma_{k}>b} l_{k} e^{-a t_{k}}\left[\sin \left(a\left(\sigma_{k}-b\right)\right)\right. \\
& \left.-i \cos \left(a\left(\sigma_{k}-b\right)\right)\right]-\frac{\pi}{a}(\sin (a(1-b))+i(1 \\
& -\cos (a(1-b)))),
\end{aligned}
$$

where separation of real and imaginary parts readily gives equalities

$$
\begin{aligned}
& \int_{0}^{\infty} e^{-a t} \ln |\varsigma(b+i t)| d t-\int_{0}^{\infty} \sin (a x) \ln |\varsigma(b+x)| d x \\
& =\frac{2 \pi}{a} \sum_{\rho, t_{k}>0, \sigma_{k}>b} l_{k} e^{-a t_{k}} \sin \left(a\left(\sigma_{k}-b\right)\right) \\
& -\frac{\pi}{a} \sin (a(1-b)) \\
& \int_{0}^{\infty} e^{-a t} \arg (\varsigma(b+i t)) d t \\
& +\int_{0}^{\infty} \cos (a x) \ln |\varsigma(b+x)| d x \\
& =-\frac{2 \pi}{a} \sum_{\rho, t_{k}>0, \sigma_{k}>b} l_{k} e^{-a t_{k}} \cos \left(a\left(\sigma_{k}-b\right)\right) \\
& -\frac{\pi}{a}(1-\cos (a(1-b))) .
\end{aligned}
$$

Here the argument is a continuous function along the contour (of course, provided there are no Riemann zeroes lying exactly on it) and the following initial values of the argument should be taken: since we have the simple pole at $z=1$ on the contour, we select for real $x>1 \arg (\varsigma(x))=0$ and for $1>b>-2 \arg (\varsigma(b+i \varepsilon))=-\pi$ where real positive $\varepsilon \rightarrow 0$.

Equations (4) and (5) are our starting point, and their first application is the following theorem.

Theorem 2. Equalities

$$
\begin{aligned}
& \int_{0}^{\infty} e^{-a t} \ln |\varsigma(b+i t)| d t \\
& \quad-\int_{0}^{\infty} \sin (a x) \ln |\varsigma(b+x)| d x+\frac{\pi}{a} \sin (a(1-b)) \\
& \quad=0 \\
& \int_{0}^{\infty} e^{-a t} \arg (\varsigma(b+i t)) d t \\
& +\int_{0}^{\infty} \cos (a x) \ln |\varsigma(b+x)| d x \\
& +\frac{\pi}{a}(1-\cos (a(1-b)))=0,
\end{aligned}
$$

where $b$, a are real positive numbers such that $1>b \geq 1 / 2$, $a(1-b) \leq \pi$ for (6) and $a(1-b) \leq \pi / 2$ for (7), hold true for some $b$ if and only if there are no Riemann function zeroes with $\sigma>b$. For $b=1 / 2$ these equalities are equivalent to the Riemann hypothesis.

Proof. For all possible Riemann $\varsigma$-function zeroes with $\operatorname{Re} \rho=$ $\sigma_{k}>b$ and hence lying inside the contour, from our choice of $b$ and the known properties of the Riemann function zeroes, we have $0<\sigma_{k}-b<1-b \leq 1 / 2$. Then the imposed conditions $a(1-b) \leq \pi$ and $a(1-b) \leq \pi / 2$ guaranty the same signs of all 
possible contributions $(2 \pi / a) \sum_{\rho, t_{k}>0, \sigma_{k}>b} l_{k} e^{-a t_{k}} \sin \left(a\left(\sigma_{k}-b\right)\right)$ and $-(2 \pi / a) \sum_{\rho, t_{k}>0, \sigma_{k}>b} l_{k} e^{-a t_{k}} \cos \left(a\left(\sigma_{k}-b\right)\right)$ from (4) and (5) and also prevent them from being equal to zero.

In (6), it is interesting for $b=1 / 2$ to take $a=2 \pi$ thus eliminating any problem with the integration over real axis (pole of the Riemann function coincides with zero of sine):

$$
\begin{aligned}
\int_{0}^{\infty} e^{-2 \pi t} \ln \left|\varsigma\left(\frac{1}{2}+i t\right)\right| d t \\
\quad-\int_{0}^{\infty} \sin (2 \pi x) \ln \left|\varsigma\left(\frac{1}{2}+x\right)\right| d x=0 .
\end{aligned}
$$

Similarly, for (7), the choice $a=\pi$ is useful:

$$
\begin{aligned}
& \int_{0}^{\infty} e^{-\pi t} \arg \left(\varsigma\left(\frac{1}{2}+i t\right)\right) d t \\
& \quad+\int_{0}^{\infty} \cos (\pi x) \ln \left|\varsigma\left(\frac{1}{2}+x\right)\right| d x+1=0 .
\end{aligned}
$$

Equalities (6a), (7a) are equivalent to the Riemann hypothesis. They have been tested numerically using the standard procedure Integrate in Mathematica. Because the first integrand here decays very fast, we can limit the integration interval of the variable $t$ to $[0,50]$. We need also to reduce the working precision (set to 50) and maximal recursion (set to 70). For the second integral in (6a) and (7a) we set the integration interval for the variable $x$ to $[0,200]$ and set the working precision and maximal recursion to 50. After 1.5 hours of CPU time we obtained for (6a) the result of $8.8044282 \times 10^{-32}$ and for (7a) after more than 8 hours $1.14767406 \times 10^{-31}$. So in both cases we have a correspondence for at least the first 30 figures.

Next, two similar theorems can be proved when we select left contour border line $(b, b+i \infty)$ lying to the left to the critical line.

\section{Theorem 2a. Equality}

$$
\begin{aligned}
& \int_{0}^{\infty} e^{-a t} \ln |\varsigma(b+i t)| d t \\
& \quad-\int_{0}^{\infty} \sin (a x) \ln |\varsigma(b+x)| d x+\frac{\pi}{a} \sin (a(1-b)) \\
& \quad=0
\end{aligned}
$$

where $a, b$ are real positive numbers such that $1 / 4 \geq b>0$ and $a=\pi n /(1 / 2-b)$, where $n$ is a positive integer such that $n \leq 1 / 2 b-1$, holds true for some $b$ if and only if there are no Riemann function zeroes with $\sigma \leq b$.

Proof. In conditions of the theorem, all contributions of the Riemann function zeroes lying on the critical line, $(2 \pi / a) \sum_{\rho, t_{k}>0, \sigma_{k}>b} l_{k} e^{-a t_{k}} \sin (a(1 / 2-b))$, to the contour integral value are equal to zero. If there are a pair of Riemann function zeroes $\rho=\sigma_{k}+i t_{k}, \rho=1-\sigma_{k}+i t_{k}$ with $1 / 2>\sigma_{k}>b$, both of them lie inside the contour and their contributions exactly compensate each other thus contributing nothing to the contour integral value. If there is a zero with $\sigma_{k} \leq b$, it lies outside the contour or on its border and hence contributes nothing while its partner zero $1-\sigma_{k}+i t_{k}$ lies inside the contour and contributes $(2 \pi / a) l_{k} \sin \left(a\left(1-\sigma_{k}-b\right)\right)$. In the conditions of the theorem, contributions of all such zeroes are nonzero and have the same sign.

Quite similarly, we have Theorem $2 \mathrm{~b}$ whose proof one-toone follows that of Theorem $2 \mathrm{a}$.

Theorem 2b. Equality

$$
\begin{aligned}
\int_{0}^{\infty} e^{-a t} \arg (\varsigma(b+i t)) d t \\
\quad+\int_{0}^{\infty} \cos (a x) \ln |\varsigma(b+x)| d x \\
+\frac{\pi}{a}(1-\cos (a(1-b)))=0,
\end{aligned}
$$

where $a, b$ are real positive numbers such that $1 / 4 \geq b>0$ and $a=\pi(2 n+1) /(1-2 b)$, where $n$ is a positive integer or zero such that $n \leq 1 / 4 b-1$, holds true for some $b$ if and only if there are no Riemann function zeroes with $\sigma \leq b$.

Unfortunately, similar theorems cannot be formulated for $1 / 2>b>1 / 4$ because no one value of $a$ can ensure the same sign of all Riemann zeroes nonlying on the critical line contributions for such a case.

\section{Rigorous Bound for the Contribution of Riemann Zeroes Not Lying on the Critical Line}

Exponential weight function appearing in the integrals considered in the previous section makes the problem of estimation of the maximal possible contribution of remaining Riemann function zeroes nonlying on the critical line a rather simple one. For example, for the real part, we know ((4) for $b=1 / 2)$ that

$$
\begin{aligned}
& \int_{0}^{\infty} e^{-a t} \ln \left|\varsigma\left(\frac{1}{2}+i t\right)\right| d t \\
& \quad-\int_{0}^{\infty} \sin (a x) \ln \left|\varsigma\left(\frac{1}{2}+x\right)\right| d x+\frac{\pi}{a} \sin \left(\frac{a}{2}\right) \\
& =\frac{2 \pi}{a} \sum_{\rho, t_{k}>0} l_{k} e^{-a t_{k}} \sin a\left(\sigma_{k}-\frac{1}{2}\right),
\end{aligned}
$$

where the sum is over all zeroes nonlying on the critical line and having $\sigma>1 / 2$. How large the r.h.s. here can be? First, we have that $(2 \pi / a)\left|\sum_{\rho, t_{k}>0} l_{k} e^{-a t_{k}} \sin a\left(\sigma_{k}-1 / 2\right)\right| \leq$ $(2 \pi / a) \sum_{\rho, t_{k}>0} l_{k} e^{-a t_{k}}$. This last sum is given by Stieltjes integral $I=(2 \pi / a) \int_{0}^{\infty} e^{-a t} d N(t)$, where $N(t)$ is a discontinuous counting function which counts the number of Riemann zeroes having the real part $\sigma_{k}>1 / 2$ and $T \geq t_{k}>0$ taking into account the order of zeroes. The integration by parts readily gives $I=\left(2 \pi / a^{2}\right) \int_{0}^{\infty} e^{-a t} N(t) d t$. About the function $N(t)$ we know that it is equal to zero when $t<T=3.3 \cdot 10^{9}$ [9]. Further, when $t>T$ it cannot exceed the function 
$\widetilde{N}(t)-\widetilde{N}(T)$, where $\widetilde{N}(t)$ is a usual zero counting function which counts zeroes in the strip $1>\sigma_{k}>0, T \geq t_{k}>0(N(t)$ is equal to $\widetilde{N}(t)-\widetilde{N}(T)$ if all zeroes with $t>T$ do not lie on the critical line). Thus the contribution at question cannot exceed

$$
I=\frac{\pi}{a^{2}} \int_{T}^{\infty} e^{-a t}(\widetilde{N}(t)-\widetilde{N}(T)) d t
$$

Now for the function $\widetilde{N}(t)$ we use the known formula

$$
\widetilde{N}(t)=\frac{t}{2 \pi}\left(\ln \frac{t}{2 \pi}-1\right)+\frac{7}{8}+Q(t)
$$

with the estimation of the reminder given by Backlund in 1916 [10]:

$$
|Q(t)|<0.137 \ln t+0.443 \ln \ln t+4.350 \text {. }
$$

Then integration in (11) with (12) and (13) is trivial and we obtain that

$$
\begin{aligned}
|I|< & \frac{1}{2 a^{3}}\left(2 \pi \cdot 0.137 \ln T+\frac{1}{a} \ln T+2 \pi \cdot 4.35\right) e^{-a T} \\
+ & \frac{2 \pi \cdot 0.443}{2 a^{3}} \int_{T}^{\infty} \frac{e^{-a t}}{\ln ^{2} t} d t+\left(\frac{2 \pi \cdot 0.137}{2 a^{3}}+\frac{1}{2 a^{5}}\right) \\
& \cdot \int_{T}^{\infty} \frac{e^{-a t}}{t} d t .
\end{aligned}
$$

Of course, $\int_{T}^{\infty}\left(e^{-a t} / \ln ^{2} t\right) d t \quad<\quad(1 / a)\left(e^{-a T} / \ln ^{2} T\right)$, $\int_{T}^{\infty}\left(e^{-a t} / t\right) d t<(1 / a)\left(e^{-a T} / T\right)$ and thus

$$
\begin{aligned}
|I| & <\frac{1}{2 a^{3}}\left(0.861 \ln T+\frac{1}{a} \ln T+27.332+\frac{2.784}{a \ln ^{2} T}\right. \\
& \left.+\frac{0.861}{a T}+\frac{1}{a^{3} T}\right) e^{-a T} .
\end{aligned}
$$

Thus for, say, $a=1$ we obtain $|I|<34.1 e^{-3.3 \cdot 10^{9}}=$ $34.1 / 10^{1.433 \cdot 10^{9}}$, and taking the maximal possible value of $a$, $a=2 \pi$, we get $|I|<0.101 e^{-2.074 \cdot 10^{10}}=0.101 / 10^{0.9 \cdot 10^{10}}$, that is, a precision with at least 1.43 milliard decimals in the first case and of 9 milliards of decimals in the second case.

To summarize, we may say that, combining a rigorous analytical treatment with the known numerical results on the Riemann function zeros on the critical line, we have found an equation of the form $A=B+\varepsilon$, where $\varepsilon=0$ is equivalent to the RH. We were able to set a bound to $\varepsilon$ of the order 1 over the nine milliardth power of 10; in this tiny factor lies the truth of the $\mathrm{RH}$. In a numerical experiment we verified the equation up to 30 decimals.

Remark 3. There is a more recent calculation of Gourdon where it is reported that the first $k=10^{13}$ Riemann zeroes are located on the critical line, but we were unable to get an exact value of $T$ from the corresponding reference [11]. See also quite recent Platt paper [12] and references cited therein for a short review of the problem.

It is also worthwhile to note that if we put the question what is the attainable precision of equalities pertinent to check up whether there are no Riemann function zeroes with $\sigma<b<1 / 2$, such a precision can be much larger because for such case in the conditions of Theorems $2,2 \mathrm{a}$, and $2 \mathrm{~b}$ much larger values of $a$ can be taken; for example, for $b=1 / 16$, we are able to use the weight function $e^{-8 \pi t}$.

\section{On Some Fourier Transforms of Logarithm of the Riemann Zeta-Function Taken along the Real Axis and Their Relation with the Riemann Hypothesis}

We now discuss what is the situation with $b<0$, that is, if we move the left border of the contour further to the left. Let for a moment still $b>-2$. First, we select $a$ in such a manner that $\sin (a(1 / 2-b))= \pm 1, a \leq \pi$. In doing so, when speaking about integral equalities involving $\int_{0}^{\infty} e^{-a t} \ln \mid \varsigma(b+$ it) $\mid d t$, we set all contributions of the Riemann function zeroes lying on the critical line to the "maximal by modulus" value $\pm(2 \pi / a) \sum_{\rho: t_{k}>0, \sigma_{k}=1 / 2} l_{k} e^{-a t_{k}}$. If we somehow know all ordinates $t_{k}$ of the Riemann function zeroes $\rho_{k}=\sigma_{k}+i t_{k}$, we are in a position to calculate the value $\Sigma_{a, \mathrm{RH}}=(2 \pi / a) \sum_{\rho: t_{k}>0} l_{k} e^{-a t_{k}}$ and then we would be able to formulate a criterion equivalent to the Riemann hypothesis of the type " $R H$ holds true if and only if the integral equality $\int_{0}^{\infty} e^{-a t} \ln |\varsigma(b+i t)| d t-$ $\int_{0}^{\infty} \sin (a x) \ln |\varsigma(b+x)| d x+(\pi / a) \sin (a(1-b))=\Sigma_{a, \mathrm{RH}}$ holds" (evident changes taking into account an appearance of trivial zeroes on the contour border are to be made if $b<-2$; see below). This is clear because, from the formula expressing the contribution of zeroes to the contour integral value $\Sigma_{a}=(2 \pi / a) \sum_{\rho, t_{k}>0, \sigma_{k}>b} l_{k} e^{-a t_{k}} \sin \left(a\left(\sigma_{k}-b\right)\right)$ and our choice of $a, b$, it immediately follows that, for zeroes with $\sigma_{k} \neq 1 / 2$, all contributions have the same sign and the module of contribution is smaller than it would be for zeroes with the same ordinates but with $\sigma_{k}=1 / 2, \mid \sin \left(a\left(\sigma_{k}-b\right)\right)+\sin (a(1-$ $\left.\left.\sigma_{k}-b\right)\right)|<| 2 \sin (a(1 / 2-b)) \mid=2$. Similar situation takes place for an integral involving an argument of the Riemann function if we select $\cos (a(1 / 2-b))= \pm 1, a \leq \pi$.

Unfortunately, we do not see how the constant $\Sigma_{a, \mathrm{RH}}$ can be efficiently calculated.

The other way around, we can select $a$ in such a manner that the condition $\cos (a(1 / 2-b))=0$ holds. Then for all such values of $a$ we obtain unconditionally (i.e., independent on the $\mathrm{RH}$ ) true equalities: all terms $-(2 \pi / a) \sum_{\rho, t_{k}>0, \sigma_{k}>b} l_{k} e^{-a t_{k}} \cos \left(a\left(\sigma_{k}-b\right)\right)$ in (5) vanish; they are either equal to zero, if $\sigma_{k}=1 / 2$, or mutually compensate each other for a pair of zeroes having $\sigma_{k}=1 / 2 \pm \alpha_{k} \neq 1 / 2$. Similar unconditional integral equalities can be obtained, starting from (4), for integrals involving logarithm of the module of the Riemann function if we select $\sin (a(1 / 2-b))=$ 0 . For completeness, we present these results as the following simple theorem. 
Theorem 4. For any real positive $a$ and any real $b$ such that $-2<b \leq 0$, one has an integral equality

$$
\begin{aligned}
\int_{0}^{\infty} e^{-a t} \arg (\varsigma(b+i t)) d t \\
\quad+\int_{0}^{\infty} \cos (a x) \ln |\varsigma(b+x)| d x \\
=-\frac{\pi}{a}(1-\cos (a(1-b)))
\end{aligned}
$$

if $\cos (a(1 / 2-b))=0$, and integral equality

$$
\begin{aligned}
\int_{0}^{\infty} e^{-a t} \ln |\varsigma(b+i t)| d t \\
\quad-\int_{0}^{\infty} \sin (a x) \ln |\varsigma(b+x)| d x \\
=-\frac{\pi}{a} \sin (a(1-b))
\end{aligned}
$$

if $\sin (a(1 / 2-b))=0$.

If we move the left border of the contour further to the left, then, contrary to the case $b>-2$, on the lower border of the contour we have a number of trivial zeroes at the points $-2,-4,-6, \ldots$ and $(4)$ is to be modified as

$$
\begin{aligned}
& \int_{0}^{\infty} e^{-a t} \ln |\varsigma(b+i t)| d t \\
& -\int_{0}^{\infty} \sin (a x) \ln |\varsigma(b+x)| d x \\
& =\frac{2 \pi}{a} \sum_{\rho, t_{k}>0, \sigma_{k}>b} l_{k} e^{-a t_{k}} \sin \left(a\left(\sigma_{k}-b\right)\right) \\
& \quad-\frac{\pi}{a} \sin (a(1-b))+\frac{\pi}{a} \sum_{k=1}^{k<[-b / 2]} \sin (a(-2 k-b)) .
\end{aligned}
$$

Correspondingly, (5) now reads

$$
\begin{aligned}
\int_{0}^{\infty} e^{-a t} \arg (\varsigma(b+i t)) d t \\
\quad+\frac{2 \pi}{a} \sum_{\rho, t_{k}>0} l_{k} e^{-a t_{k}} \cos \left(a\left(\sigma_{k}-b\right)\right) \\
+\int_{0}^{\infty} \cos (a x) \ln |\varsigma(b+x)| d x \\
+\frac{\pi}{a}(1-\cos (a(1-b))) \\
\quad-\frac{\pi}{a} \sum_{k=1}^{k<[-b / 2]}(1-\cos (a(-2 k-b)))=0 .
\end{aligned}
$$

In this last equality we start from the following initial value of an argument function: $\arg (\varsigma(b+i \varepsilon))=-\pi+[-b / 2]$. $\pi$, where real positive $\varepsilon \rightarrow 0$. (As usual, [-b/2] means the whole part of positive $-b / 2$ number.) Evident corresponding modifications should be done in Theorem 4 .

One of such unconditionally true equalities, namely, that obtained from (5) putting $a=2 \pi$ and $b=-7 / 2$, that is, $\int_{0}^{\infty} e^{-2 \pi t} \arg (\varsigma(-7 / 2+i t)) d t+\int_{0}^{\infty} \cos (2 \pi x) \ln \mid \varsigma(-7 / 2+$ $x) \mid d x=0$, has also been checked up numerically. (Note that here $b<-2$.) In this case, we calculated the first integral with the same parameters as discussed above. For the second integral we extended the integration's interval to $[0,250]$ and the maximal recursion to 100 . The result $8.74533283 \times 10^{-30}$ is similar to two previously mentioned cases. Note that here $\arg (\varsigma(-7 / 2+i \varepsilon))=0$ and the contribution of the pole exactly compensates that of the first trivial zero; hence in fact the two integrals, when summing, compensate each other up to 30 digits as found in the computations.

Interesting new possibilities appear if we take certain value of $a$ and introduce a sequence $b_{i} \rightarrow-\infty$ in such a manner that the condition $\cos \left(a\left(1 / 2-b_{i}\right)\right)=1$ holds for any $i$. Below we illustrate such approach for a particular case $a=2 \pi, b_{n}=-2 n-1 / 2$ ( $n$ is a positive integer). We have the following particular case of (19):

$$
\begin{aligned}
& \int_{0}^{\infty} e^{-2 \pi t} \arg \left(\varsigma\left(-2 n-\frac{1}{2}+i t\right)\right) d t+\Sigma_{2 \pi} \\
& \quad+\int_{0}^{\infty} \cos (2 \pi x) \ln \left|\varsigma\left(-2 n-\frac{1}{2}+x\right)\right| d x+\frac{1}{2} \\
& \quad-\frac{n}{2}=0
\end{aligned}
$$

where $\Sigma_{2 \pi}:=\sum_{\rho: t_{k}>0} l_{k} e^{-2 \pi t_{k}} \cos \left(2 \pi\left(\sigma_{k}+1 / 2\right)\right)$ is independent on $n$.

Let us now analyze both integrals of (20) starting from $\int_{0}^{\infty} e^{-2 \pi t} \arg (\varsigma(-2 n-1 / 2+i t)) d t$. All estimations below are done with the $O(1)$ precision because $O(1 / n)$ and smaller terms tend to zero for large $n$. To begin with, we should include the contribution of an initial value of an argument of the Riemann function, which is equal to $\arg (\varsigma(b+i \varepsilon))=(n-$ $1) \cdot \pi$, to $(20)$. This contributes $(n-1) / 2$ to the integral value. Now let us change $\varsigma(-2 n-1 / 2+i t)$ function to $\varsigma(2 n+3 / 2-i t)$ function using functional equation [3]:

$$
\varsigma(s)=\frac{1}{\pi}(2 \pi)^{s} \sin \frac{\pi s}{2} \Gamma(1-s) \varsigma(1-s) .
$$

Factor $(2 \pi)^{-2 n-1 / 2+i t}$ in (21) contributes $\ln (2 \pi) / 4 \pi^{2}$ to the integral value. For sine factor, taking $n$ odd, we have $\sin ((-1 / 4-n+i t / 2) \pi)=\sin ((1 / 4-i t / 2) \pi)=$ $(\sqrt{2} / 2)(-i \sinh (\pi t / 2)+\cosh (\pi t / 2))$. Its argument is $-\arctan (\tanh (\pi t / 2))$; hence we need to handle an integral $-\int_{0}^{\infty} e^{-2 \pi t} \arctan (\tanh (\pi t / 2)) d t$. Integrating by parts we see that this contribution is equal to $-1 / 2 \pi \cdot(\pi /$ 2) $\int_{0}^{\infty}\left(\mathrm{e}^{-2 \pi \mathrm{t}} / \cosh (\pi t)\right) d t=-(1 / 2) \int_{0}^{\infty}\left(e^{-2 \pi t} /\left(e^{\pi t}+e^{-\pi t}\right)\right) d t$; with the variable transform $y=e^{\pi t}$ this is easily calculated to be equal to $-1 / 2 \pi+1 / 8$.

Integral involving an argument of Riemann $\varsigma$-function tends to zero when $n \rightarrow \infty$, so next we evaluate the 
contribution of the gamma-function. We use Stirling formula [13]

$$
\begin{aligned}
\ln \Gamma\left(\frac{3}{2}+2 n-i t\right)= & (1+2 n-i t) \ln \left(\frac{3}{2}+2 n-i t\right) \\
& -\left(\frac{3}{2}+2 n-i t\right)+0.5 \ln 2 \pi \\
& +g(x),
\end{aligned}
$$

where $g(x)=\int_{0}^{\infty}\left(1 / 2-1 / t+1 /\left(e^{t}-1\right)\right)\left(e^{-t x} / t\right) d t[13]$, and have

$$
\begin{aligned}
& \operatorname{Im} \int_{0}^{\infty} e^{-2 \pi t}(1+2 n-i t) \ln \left(\frac{3}{2}+2 n-i t\right) d t \\
& =\operatorname{Im} \frac{1}{2 \pi} \int_{0}^{\infty} e^{-2 \pi t}\left(-i \ln \left(\frac{3}{2}+2 n-i t\right)\right. \\
& \left.\quad-i \frac{1+2 n-i t}{3 / 2+2 n-i t}\right) d t=-\frac{1}{4 \pi^{2}} \ln \left(\frac{3}{2}+2 n\right) \\
& \quad-\frac{1}{4 \pi^{2}}+O\left(\frac{1}{n}\right) .
\end{aligned}
$$

Further, $-\operatorname{Im} \int_{0}^{\infty} e^{-2 \pi t}(3 / 2+2 n-i t) d t=1 / 4 \pi^{2}$. Factor $0.5 \ln 2 \pi$ is real and contributes nothing. It is not difficult to see that integral involving $g(z)$ tends to zero with $n$ tending to infinity.

Now we need to analyze second integral in (20), that is, the integral $\int_{0}^{\infty} \cos (2 \pi x) \ln |\varsigma(-1 / 2-2 n+x)| d x$. With a variable change $y=-1 / 2-2 n+x$ it is equal to $-\int_{-1 / 2-2 n}^{\infty} \cos (2 \pi y) \ln |\varsigma(y)| d y$ which we divide into $-\int_{-1 / 2-2 n}^{1 / 2}-\int_{1 / 2}^{\infty}$. For the first integral we use the variable transform $x=1-y$ to write that it is equal to $-\int_{1 / 2}^{3 / 2+2 n} \cos (2 \pi x) \ln |\varsigma(1-x)| d x$ and then use the same functional equation (21) again, so we need to estimate

$$
\begin{aligned}
I_{2} & =-\int_{1 / 2}^{3 / 2+2 n} \cos (2 \pi x) \\
& \cdot \ln \left|\frac{1}{\pi}(2 \pi)^{1-x} \sin \frac{\pi(1-x)}{2} \Gamma(x) \varsigma(x)\right| d x .
\end{aligned}
$$

First, $\int_{1 / 2}^{3 / 2+2 n} \cos (2 \pi x) \ln \left|(1 / \pi)(2 \pi)^{1-x}\right| d x=0$. For an integral involving logarithm of gamma-function we again use the Stirling formula $\ln \Gamma(x)=(x-1 / 2) \ln (x)-x+0.5 \ln 2 \pi+$ $g(x)[13]$ and get

$$
\begin{aligned}
- & \int_{1 / 2}^{3 / 2+2 n} \cos (2 \pi x)\left(x-\frac{1}{2}\right) \ln (x) d x \\
= & \frac{1}{2 \pi} \int_{1 / 2}^{3 / 2+2 n} \sin (2 \pi x)\left(\ln x+1-\frac{1}{2 x}\right) d x \\
= & \frac{1}{2 \pi} \int_{1 / 2}^{3 / 2+2 n} \sin (2 \pi x)\left(\ln x-\frac{1}{2 x}\right) d x \\
= & -\frac{1}{2 \pi} \int_{1 / 2}^{3 / 2+2 n} \sin (2 \pi x) \frac{1}{2 x} d x \\
& -\left.\frac{1}{4 \pi^{2}} \cos (2 \pi x) \ln x\right|_{1 / 2} ^{3 / 2+2 n} \\
& +\frac{1}{4 \pi^{2}} \int_{1 / 2}^{3 / 2+2 n} \cos (2 \pi x) \frac{1}{x} d x \\
= & \frac{1}{4 \pi^{2}}\left(\ln \left(\frac{3}{2}+2 n\right)+2 n\right) \\
& +\frac{1}{4 \pi^{2}} \int_{1 / 2}^{3 / 2+n} \frac{1}{x} \cos (2 \pi x) d x \\
& -\frac{1}{2 \pi} \int_{1 / 2}^{3 / 2+2 n} \sin (2 \pi x) \frac{1}{2 x} d x
\end{aligned}
$$

To finish the consideration of the contribution of the logarithm of gamma-function we need to add the contribution of $g(x)$ which is $-\int_{1 / 2}^{\infty} \cos (2 \pi x) d x \int_{0}^{\infty}(1 / 2-1 / t+$ $\left.1 /\left(e^{t}-1\right)\right)\left(e^{-t x} / t\right) d t$. Using $\cos (2 \pi x)=(1 / 2)\left(e^{2 \pi x i}+e^{-2 \pi x i}\right)$ we can perform integration over $x:-\int_{1 / 2}^{\infty} \cos (2 \pi x) e^{-t x} d x=$ $e^{-t / 2}\left(t /\left(t^{2}+4 \pi^{2}\right)\right)$, and thus the contribution at question is $\int_{0}^{\infty}\left(1 / 2-1 / t+1 /\left(e^{t}-1\right)\right)\left(e^{-t / 2} /\left(t^{2}+4 \pi^{2}\right)\right) d t$.

Finally we need to analyze the contribution of the sine factor in (24) which is $-\int_{1 / 2}^{3 / 2+2 n} \cos (2 \pi x) \ln \mid \sin (\pi(1-$ $x) / 2)\left|d x=-\int_{1 / 2}^{3 / 2+2 n} \cos (2 \pi x) \ln \right| \cos (\pi x / 2) \mid d x$. Integration is to be applied with caution here due to the presence of the module. To avoid errors, it is reasonable to consider separately integrals $\int_{1 / 2}^{1}, \int_{2 n+1}^{2 n+3 / 2}=\int_{1}^{3 / 2}$, and $\int_{1}^{2}$; the latter is an integral along the period. Integrating by parts, we have

$$
\begin{aligned}
& -\int_{1 / 2}^{1} \cos (2 \pi x) \ln \left|\cos \frac{\pi x}{2}\right| d x \\
& =-\int_{1 / 2}^{1} \cos (2 \pi x) \ln \left(\cos \frac{\pi x}{2}\right) d x \\
& =-\frac{1}{4} \int_{1 / 2}^{1} \sin (2 \pi x) \tan \frac{\pi x}{2} d x,
\end{aligned}
$$


and further

$$
\begin{aligned}
- & \frac{1}{4} \int_{1 / 2}^{1} \sin (2 \pi x) \tan \frac{\pi x}{2} d x \\
& =-\int_{1 / 2}^{1} \sin ^{2} \frac{\pi x}{2} \cos (\pi x) d x \\
& =\frac{1}{2} \int_{1 / 2}^{1}(\cos (\pi x)-1) \cos (\pi x) d x \\
& =\frac{1}{2} \int_{1 / 2}^{1}\left(\frac{1+\cos (2 \pi x)}{2}-\cos (\pi x)\right) d x \\
& =\frac{1}{8}-\left.\frac{1}{2 \pi} \sin (\pi x)\right|_{1 / 2} ^{1}=\frac{1}{8}+\frac{1}{2 \pi} .
\end{aligned}
$$

Similarly, $\quad-\int_{1}^{3 / 2} \cos (2 \pi x) \ln |\cos (\pi x / 2)| d x$ $-\int_{1}^{3 / 2} \cos (2 \pi x) \ln (-\cos (\pi x / 2)) d x=1 / 8+1 / 2 \pi$ while integral over the period is equal to $1 / 4$ (use the same approach as above, or entry number 4.384 .3 of [14] with evident variable changes), so the total contribution of the sine factor in (24) to (20) is equal to $n / 2+1 / 4+1 / \pi$.

Collecting everything together we see the cancelling of $O(n)$ and $O(\ln (n))$ terms. Thus, considering the limit $n \rightarrow$ $\infty$, we have established the following relation expressing certain particular value of Fourier transform of the logarithm of the Riemann zeta-function:

$$
\begin{aligned}
\int_{1 / 2}^{\infty} \cos (2 \pi x) \ln |\varsigma(x)| d x \\
=\frac{1}{2} \Sigma_{2 \pi}+\frac{7}{16}+\frac{1}{4 \pi}+\frac{\ln (4 \pi)}{8 \pi^{2}}-\frac{c i(\pi)}{8 \pi^{2}}+\frac{s i(\pi)}{8 \pi} \\
\quad+\frac{1}{2} \int_{0}^{\infty}\left(\frac{1}{2}+\frac{1}{e^{t}-1}-\frac{1}{t}\right) \frac{e^{-t / 2}}{t^{2}+4 \pi^{2}} d t
\end{aligned}
$$

Here we used standard notation $s i(x)=-\int_{x}^{\infty}(\sin q / q) d q$, $c i(x)=-\int_{x}^{\infty}(\cos q / q) d q$ [14]. Of course, similar equalities can be established for many other such Fourier transforms.

Repeating what has been said above about the "maximality" of the sum $\Sigma_{2 \pi, \mathrm{RH}}:=\sum_{\rho: t_{k}>0} l_{k} e^{-2 \pi t_{k}}$, we again could formulate a statement of the type " $R H$ holds true if and only if the integral equality (28) with $\Sigma_{2 \pi}=\Sigma_{2 \pi, \mathrm{RH}}$ holds." Numerical calculation taking into account first 100 zeroes of the Riemann function gives the value $\Sigma_{2 \pi}=$ $2.6904854 \ldots \cdot 10^{-39}$. Equality (28) has been tested numerically and shown to be true: without the term $\Sigma_{2 \pi}$ both sides coincide up to 39 figures after decimal and are equal to $0.5620211964552684935093570395449423344 \ldots$... Then, of course, they start to differ.

\section{Conclusions}

In this paper we have established a number of new criteria involving the integrals of the logarithm of the Riemann $\varsigma^{-}$ function and equivalent to the Riemann hypothesis, this time with the exponential weight functions. Exponential weight functions lead to rather simple expressions for the contribution of the Riemann function zeroes not lying on the critical line to the contour integral value. This enabled obtaining a rigorous estimation of the possible error which was shown to be extremely small.

We also show how certain Fourier transforms of the logarithm of the Riemann zeta-function taken along the real (demi)axis are expressible via elementary functions plus logarithm of the gamma-function and definite integrals thereof, as well as certain sums over trivial and nontrivial Riemann function zeroes. In our opinion, further study of similar Fourier transforms is interesting and might be useful in the Riemann researches.

\section{Conflict of Interests}

The authors declare that there is no conflict of interests regarding the publication of this paper.

\section{References}

[1] S. K. Sekatskii, S. Beltraminelli, and D. Merlini, "On equalities involving integrals of the logarithm of the Riemann-function and equivalent to the Riemann hypothesis," Ukrainian Mathematical Journal, vol. 64, no. 2, pp. 218-228, 2012.

[2] S. Beltraminelli, D. Merlini, and S. Sekatskii, "A hidden symmetry related to the Riemann hypothesis with the primes into the critical strip," Asian-European Journal of Mathematics, vol. 3, no. 4, pp. 555-563, 2010.

[3] E. C. Titchmarsh and E. R. Heath-Brown, The Theory of the Riemann Zeta-Function, Clarendon Press, Oxford, UK, 1988.

[4] F. T. Wang, "A note on the Riemann zeta-function," Bulletin of the American Mathematical Society, vol. 52, pp. 319-321, 1946.

[5] V. V. Volchkov, "On an equality equivalent to the Riemann hypothesis," Ukrainian Mathematical Journal, vol. 47, no. 3, pp. 491-493, 1995.

[6] M. Balazard, E. Saias, and M. Yor, "Notes sur la fonction $\zeta$ de Riemann, 2," Advances in Mathematics, vol. 143, no. 2, pp. 284287, 1999.

[7] D. Merlini, "The Riemann magneton of the primes," Chaos and Complexity Letters, vol. 2, no. 1, pp. 93-98, 2006.

[8] E. C. Titchmarsh, The Theory of Functions, Oxford University Press, Oxford, UK, 1939.

[9] C. Ramaré and Y. Saouter, "Short effective intervals containing primes," Journal of Number Theory, vol. 98, no. 1, pp. 10-33, 2003.

[10] R. J. Backlund, "Über die Nullstellen der Riemannschen Zetafunktion," Acta Mathematica, vol. 41, no. 1, pp. 345-375, 1916.

[11] X. Gourdon, "The $10^{13}$ first zeroes of the Riemann Zeta Function, and zeroes computation at very large height," 2004, http://numbers.computation.free.fr/Constants/Miscellaneous/ zetazeros1e13-1e24.pdf.

[12] D. J. Platt, "Computing $\pi(x)$ analytically," Mathematics of Computation, vol. 84, no. 293, pp. 1521-1535, 2015.

[13] A. Erdelyi, Ed., Higher Transcendental Functions. Based, in Part, On Notes Left by Harry Bateman, vol. 1, McGraw-Hill, New York, NY, USA, 1953.

[14] I. S. Gradshtein and I. M. Ryzhik, Tables of Integrals, Series and Products, Academic, New York, NY, USA, 1990. 




Advances in

Operations Research

mansans

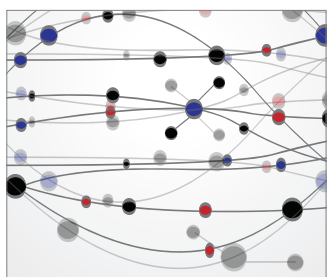

The Scientific World Journal
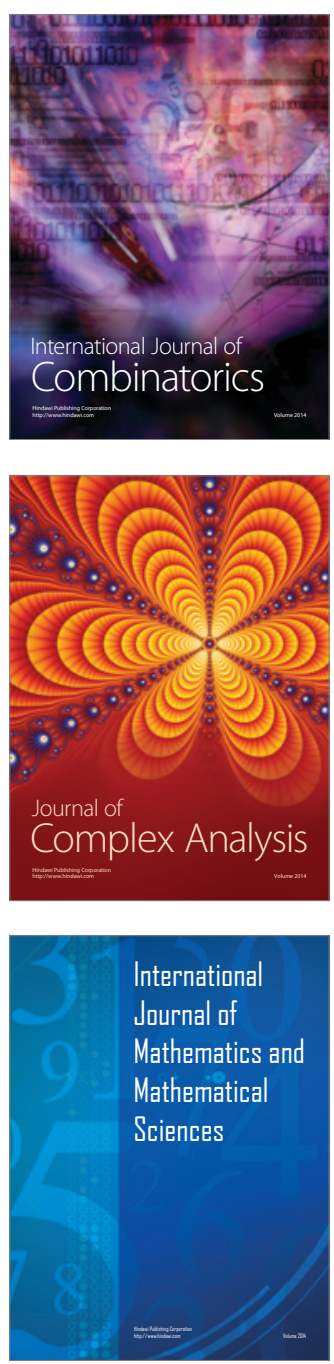


Submit your manuscripts at http://www.hindawi.com
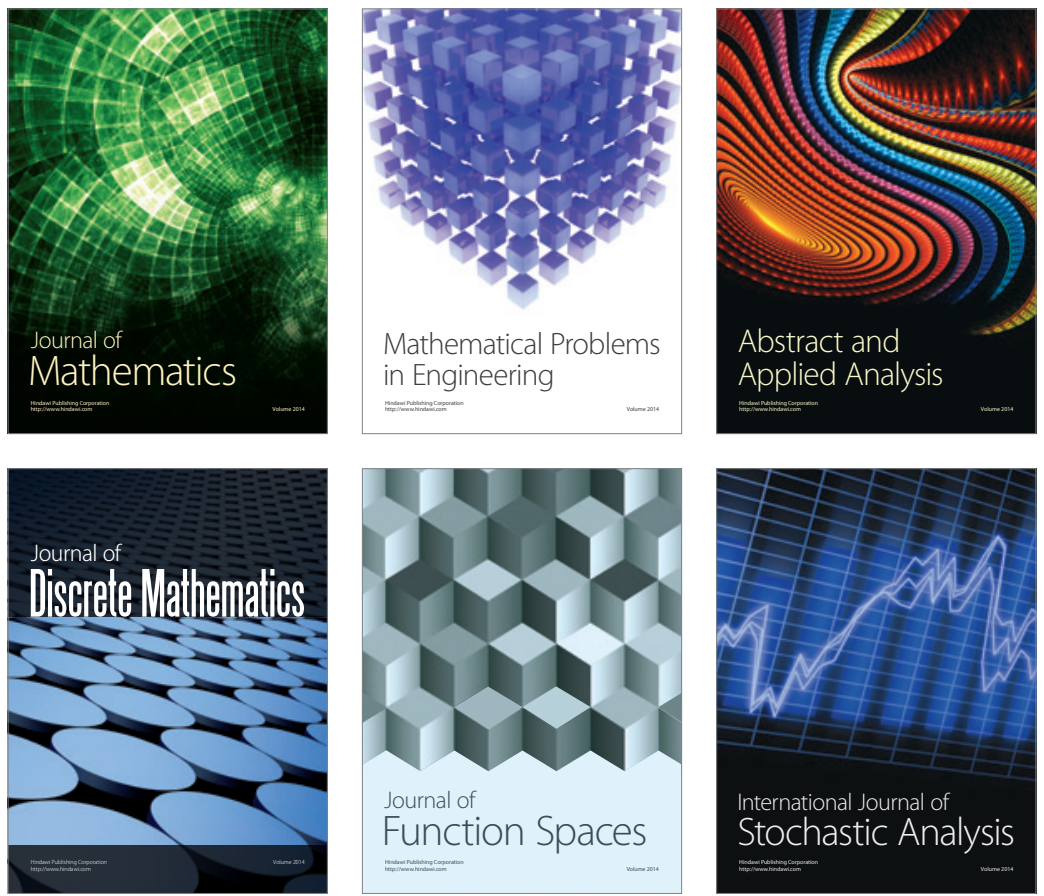

Journal of

Function Spaces

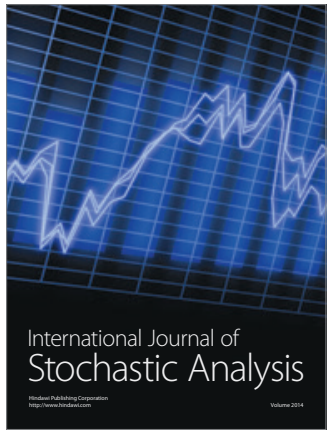

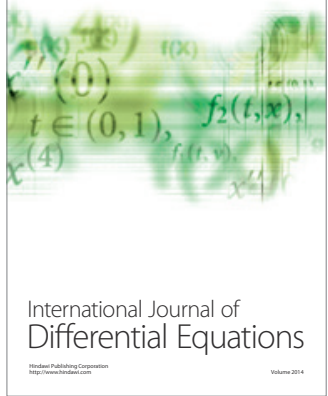
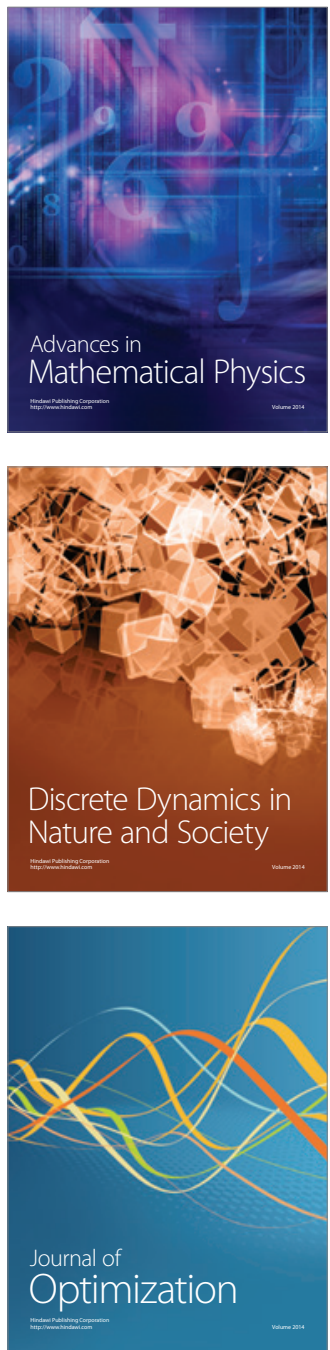